\title{
Thermal Modeling and Experimental Validation of a Large Prismatic Li-ion Battery
}

\author{
Nicolas Damay ${ }^{a, b}$, Christophe Forgez ${ }^{a *}$, Marie-Pierre Bichat ${ }^{b}$, Guy Friedrich $^{a}$, Alejandro Ospina ${ }^{a}$ \\ ${ }^{a}$ Laboratoire d'Electromécanique de Compiègne, EA 1006 \\ Université de Technologie de Compiègne, BP 20529 - 60205 Compiègne Cedex, FRANCE \\ ${ }^{b}$ E4V, 9 avenue Georges Auric, 72000 Le Mans, FRANCE \\ * : corresponding author (christophe.forgez@utc.fr)
}

\begin{abstract}
This paper deals with the thermal modeling and experimental validation of a large prismatic Li-ion battery. A lumped model representing the main thermal phenomena in the cell, in and outside the casing is proposed. Most of the parameters are determined analytically, using physical and geometrical properties. The heat capacity, the internal thermal resistance and interfacial thermal resistance between the cell and its cooling system are experimentally identified. The proposed model is validated with a precision of $1^{\circ} \mathrm{C}$.
\end{abstract}

Keywords-Batteries, Li-ion, Thermal Modeling, System Identification

\section{INTRODUCTION}

Energy management and security are key issues for electric and hybrid vehicles development. Battery management systems (BMS) have been developed to ensure these goals. Inside a BMS, the thermal management has an essential role in the operating system supervision [1]. Furthermore, having a thermal model able to estimate the internal temperature of the battery under operation can help improving the accuracy of an electrical model used in the BMS [2], avoid exceeding the battery thermal limitations [3] or allow a more efficient energy management.

Several papers deal with thermal modeling of battery, using different approaches such as Partial Differential Equation (PDE) model [4], Linear Parameter-Varying (LPV) model [5], finite element model [6] or lumped parameter model [7]. In most of them, a thermal model is coupled with an electrochemical model so as to simulate the temperature profile of a battery under different operating conditions, geometries or cooling rate. Such coupled models can also be used to explore pulse power limitations, in order to prevent thermal runaway or to design heat dissipation systems. Three-dimensional thermal models have also been investigated in order to provide a better insight about the behavior of cells under abuse conditions. Such models based on finite element model are well-suited for the battery design purpose, but are not compatible with the low computational resources of micro-controllers used in BMS.

A thermal model of a large prismatic $\mathrm{Li}$-ion cell $\left(\mathrm{LiFePO}_{4}\right)$ based on a lumped electric equivalent model is presented in this paper. It aims to represent a cell thermal behavior inside a battery pack. Such a model is able to estimate the internal temperature of a cell, and simple enough to be implemented in real time calculators.

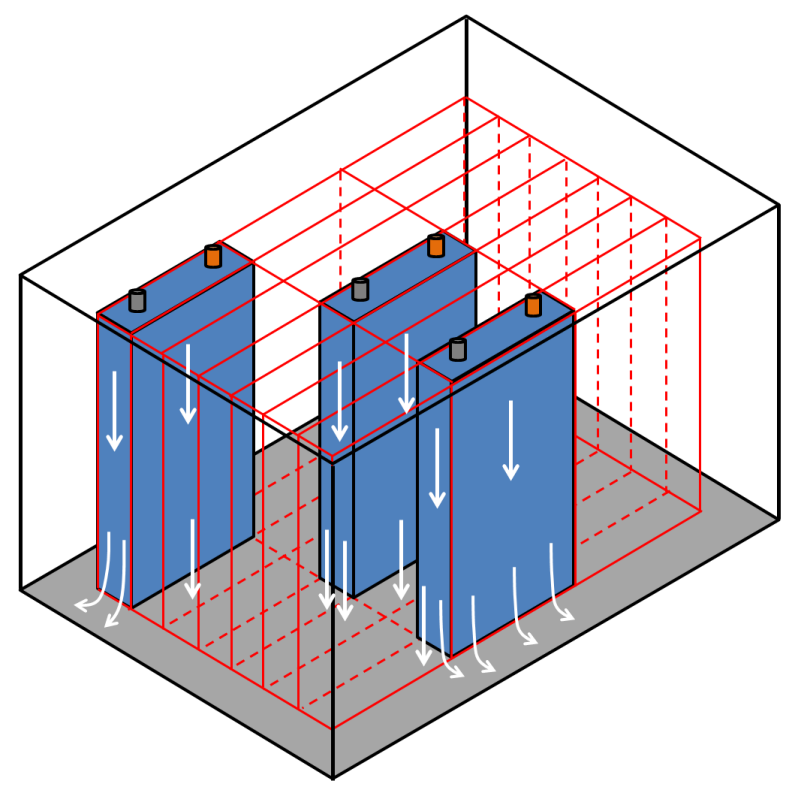

Figure 1. Prismatic cells $(3 \times 7)$ integration in a pack

Inside of a prismatic battery pack, cell temperatures have been measured as quite homogeneous and hottest elements appeared to be located in central location. For the latter, surrounding cells are almost at the same temperature and their heat is so evacuated through their bottom faces (see Fig. 1). As a result, their cooling performances are strongly dependent on the interfacial thermal resistance between the bottom face and the cooling system. In order to analyze the efficiency of the cooling system, this resistance must be evaluated. Because it has no thickness, it can not be measured using sensors and, consequently, must be identified indirectly.

A cell (core and casing) and its environment have been represented by a lumped model. Most of the thermal parameters of this model are determined analytically, using physical and geometrical properties of the materials. On the contrary, heat capacity, internal resistance and interfacial resistance between the casing and its cooling system are experimentally identified.

In a first part, main thermal phenomena are modeled [7], [8]. In a second part, analytical and experimental methods for thermal parameters identification are presented [2], [9]. Finally, experimental validation of the proposed model through a discharge-charge cycle is presented and discussed. 


\section{THERMAL MODEL}

We used a lumped thermal model [2], [8] - also called equivalent electric circuit - to model our cell. This approach is based on the formal analogy between thermal and electrical phenomena. Nodes are associated with volumes (assumed isothermal), capacitances represent heat accumulation, resistances represent heat transfers (by conduction, convection or radiation), current sources represent heat generation and voltage sources represent set temperatures. Capacitances, current and voltage sources are used between a node and the ground node. To lighten the model representation, voltage sources are replaced by temperatures they set.

In our application, cells have been integrated in a battery pack as shown by figure 1 . Cells are connected to a cooling system by their bottom faces. Heat flows to the cooling system are represented by white arrows. As for the horizontal heat transfers, 3 configurations with different boundary conditions can be highlighted:

- at the center: heat may flow between cells by conduction;

- on a side: heat can also flow between the cell and the pack inner atmosphere by convection through one face;

- on a corner: heat can also flow between the cell and the pack inner atmosphere by convection through two faces.

The cell was modeled by the thermal network shown in figure 2 where there is one central node for the cell core, one node per face and one per terminal. Main thermal phenomena in the cell (core), in the aluminum casing and outside the casing have been modeled.

In the core: Green elements stand for internal phenomena (heat generation, accumulation and transfers). Because of the poor thermal contacts between them, heat transfers between the cell core and faces in $\mathrm{y}$ and $\mathrm{z}$ directions are neglected. Due to the foil stacking internal structure, we considered the cell core having a homogeneous specific heat and an anisotropic thermal conductivity (being equal in $\mathrm{y}$ and $\mathrm{z}$ directions and different in the $\mathrm{x}$ direction) [9]. Despite its dimensions, the cell core is considered isothermal in $\mathrm{y}$ and $\mathrm{z}$ directions thanks to the current collectors. This has been confirmed by tests made by a third party. All core thermal properties are assumed to be constant regarding the state of charge (SoC) [10]. As for $R_{i,+}$ and $R_{i,-}$, they stand for conduction through the current collectors.

In the core: Green elements stand for internal phenomena (heat generation, accumulation and transfers). It can be seen that heat transfers between the cell core and faces in $y$ and $z$ directions are neglected. This is due to poor thermal contacts between them. Due to the foil stacking internal structure, we considered the cell core having a homogeneous specific heat and an anisotropic thermal conductivity (being equal in $\mathrm{y}$ and $\mathrm{z}$ directions and different in the $\mathrm{x}$ direction) [9]. Despite its dimensions, the cell core is considered isothermal in $y$ and $\mathrm{Z}$ directions thanks to the current collectors. This has been confirmed by tests made by a third party. All core thermal properties are assumed to be constant regarding the state of

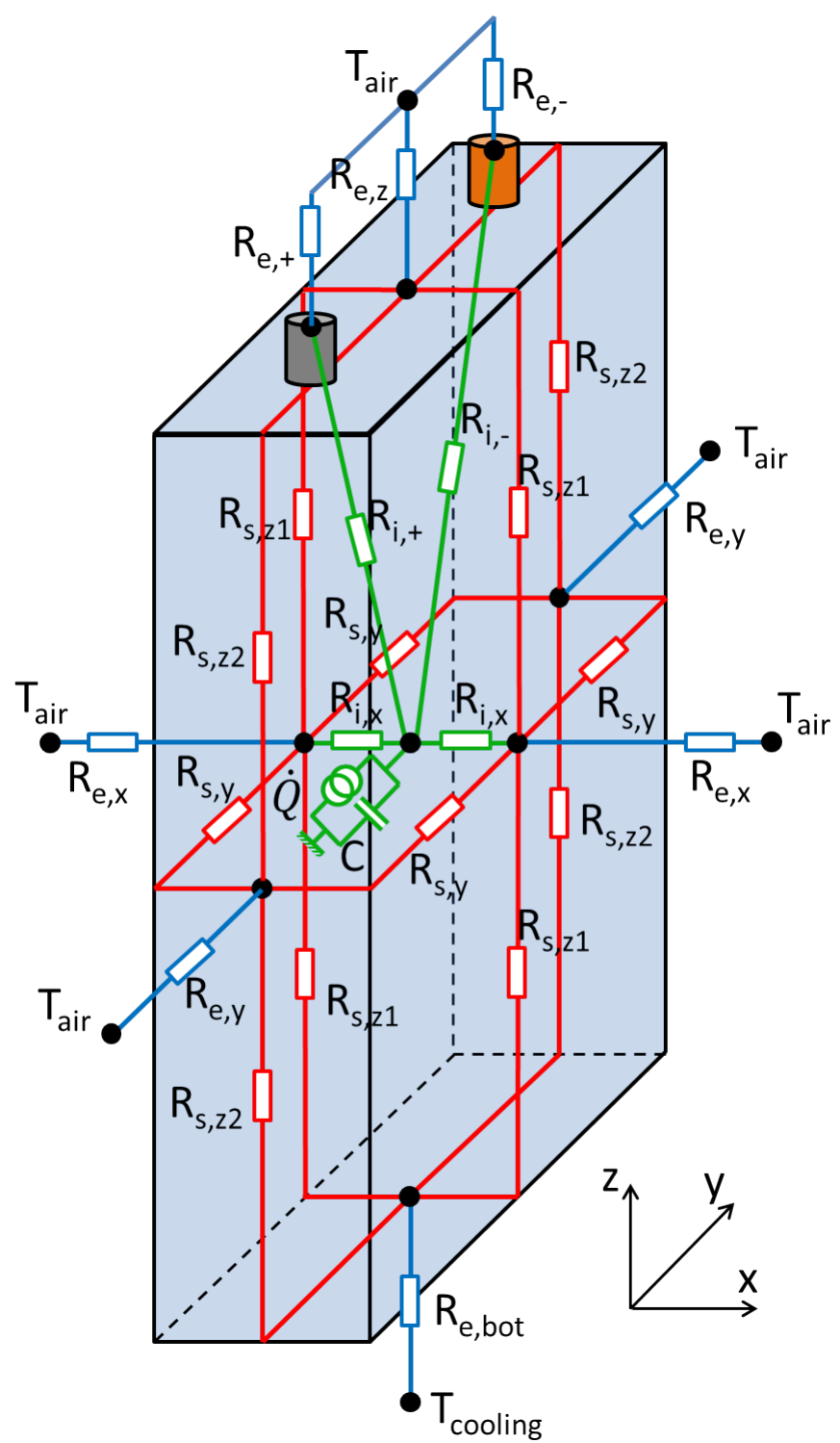

Figure 2. Lumped thermal model of a cell.

charge (SoC) [10]. As for $R_{i,+}$ and $R_{i,-}$, they stand for conduction through the current collectors.

In the casing: Red elements stand for heat transfers through the casing. All casing thermal capacities are neglected compared to the core thermal capacity and no heat generation has been considered. Consequently, there are only heat transfers by conduction through the casing.

Outside the casing: Blue elements represent thermal exchanges between the cell and its environment (by conduction, convection and/or radiation, depending on the cell location in the pack). The resistance $R_{e, b o t}$ below the cell represents the contact resistance between the cell bottom and the cooling system. Other blue resistances represent heat exchanges, by the lateral faces and by the top face, either with the ambient air or with other cells. 

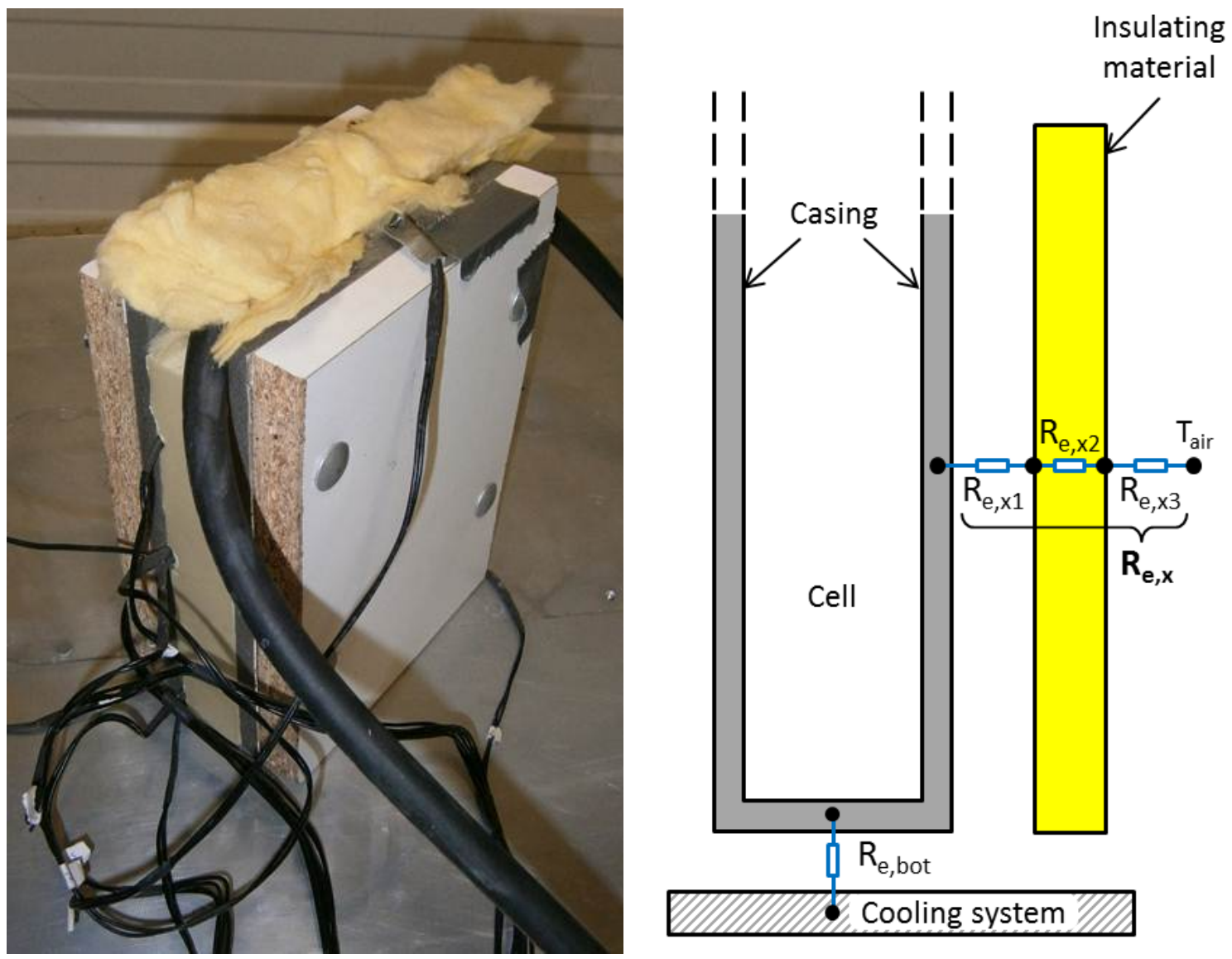

Figure 3. (a) Experimental setup approaching pack central cell conditions and (b) details about the heat transfer resistances outside the casing.

\section{PARAMETERS CHARACTERIZATION}

In order to simplify the model identification, we have considered a cell located in the center of a pack. In this case, heat flows mostly in the $\mathrm{z}$ direction, because surrounding cells are supposed to be at the same temperature. Those conditions have been experienced by packing a cell with insulating materials and placing it on a temperature-regulated system (Fig. 3a).

Values and determination modes of the different components of our thermal model are summarized in the table I. Parameters have been sorted by location and by type.

Heat generation: We chose to use the cell internal heat generation to characterize its thermal parameters. The heat generation $\dot{Q}$ has been modeled by equation (1) [2]. The first term stands for resistive dissipation and is always positive (irreversible heat). The second term is the entropic heat and can be positive or negative (reversible heat) [11].

$$
\dot{Q}=I_{\text {cell }}\left(V_{\text {cell }}-V_{\text {ocv }}\right)+I_{\text {cell }} T_{\text {cell }} \frac{\partial V_{o c v}}{\partial T}
$$

The irreversible heat can be calculated :

- $\quad$ if the open circuit voltage $V_{o c v}$ is known. It depends on the State of Charge (SoC), on the current direction (following a hysteresis cycle, which is about $10 \mathrm{mV}$ large) [12] and on the temperature (about $0.1 \mathrm{mV} / \mathrm{K}$ );

\begin{tabular}{|c|c|c|c|}
\hline & Value & Evaluation & Equation \\
\hline$R_{i, x}$ & $0.8 K \cdot W^{-1}$ & Experimental & $(3)$ \\
\hline$R_{i,+}$ & $4.3 K \cdot W^{-1}$ & Analytical & $(4)$ \\
\hline$R_{i,-}$ & $4.7 K \cdot W^{-1}$ & Analytical & $(4)$ \\
\hline \hline$R_{s, y}$ & $3.1 K \cdot W^{-1}$ & Analytical & $(4)$ \\
\hline$R_{s, z 1}$ & $8.9 K \cdot W^{-1}$ & Analytical & $(4)$ \\
\hline$R_{s, z 2}$ & $36.9 K \cdot W^{-1}$ & Analytical & $(4)$ \\
\hline \hline$R_{e, x}$ & $24.3 K \cdot W^{-1}$ & Analytical & $(4)$ \\
\hline$R_{e, y}$ & $55.0 K \cdot W^{-1}$ & Analytical & $(4)$ \\
\hline$R_{e, z}$ & $6.7 K \cdot W^{-1}$ & Analytical & $(4)$ \\
\hline$R_{e,+/-}$ & $10.6 K \cdot W^{-1}$ & Analytical & \\
\hline$R_{e, b o t}$ & $1.8 K \cdot W^{-1}$ & Experimental & \\
\hline \hline$\dot{Q}$ & Variable & Estimation & $(1)$ \\
\hline \hline$C$ & $1000 J \cdot K^{-1}$ & Experimental & $(2)$ \\
\hline
\end{tabular}

Table I. VALUES AND DETERMINATION MODES OF THE DIFFERENT COMPONENTS OF OUR THERMAL MODEL

- $\quad$ by measuring the cell voltage $V_{\text {cell }}$, which depends on the current $I_{c e l l}$, on time, on cell temperature $T_{\text {cell }}$, on the SoC and on ageing;

- $\quad$ by measuring the current $I_{\text {cell }}$.

The second term is evaluated in the same way, given the knowledge of the term $\frac{\partial V_{o c v}}{\partial T}$ (which only depends on the SoC) and by measuring $T_{\text {cell }}$ and $I_{\text {cell }}$.

Values of $V_{o c v}$ and $\frac{\partial V_{o c v}}{\partial T}$ have been evaluated during specific tests [2]. 
Heat capacity: In order to identify the heat capacity $C$, the battery was packed with insulating materials on its different faces (including the bottom's). It was heated by imposing a $+/-1 C$ square current with a period of 20 s (a 1C current fully discharges the battery in $1 \mathrm{~h}$ ). In these conditions, the internal temperature was increasing as a ramp at the beginning of the test. Consequently, the heat capacity was determined with equation (2).

This current solicitation has been chosen because it keeps the cell SoC constant. As a result, $V_{o c v}$ is well-known, because it can be measured before beginning the test. Besides, the square profile eliminates the irreversible heat. Finally, a 20second-long period is very small compared to the thermal time constant of the cell. Thus, the average heat generation $\dot{Q}_{a v g}$ can be used directly in the calculus.

$$
C=\frac{\dot{Q}_{a v g}}{d T_{\text {cell }} / d t}
$$

This heat capacity value corresponds to a specific heat of $0.83 \mathrm{~J} \cdot \mathrm{g}^{-1} \cdot \mathrm{K}^{-1}$, which is consistent with results found in literature.

Internal heat transfer in $\mathbf{x}$ direction: The cell internal resistance $R_{i, x}$ was experimentally obtained using the value of $C$ and measurements made by a third party. Several thermocouples have been put inside (in its median plan) and outside a cell. The cell has been placed in a climatic chamber with no insulating material, heated by a full discharge and then rested. During the cell cooling, most of its heat was evacuated by its faces in $\mathrm{x}$ direction. As a result, it was possible to derive formula (3) from the thermal model.

$$
R_{i, x}=\frac{\Delta T}{C \frac{d T_{i n} / d t}{2}}=0.8 K . W^{-1}
$$

$\Delta T$ is the temperature difference between the cell core and the face in $\mathrm{x}$ direction. The heat flow running through $R_{i, x}$ is determined by the heat capacity "discharge".

Internal heat transfer toward terminals: Transfers between the core and the terminals were analytically determined using their geometric features and formula (4) [13], where $L$ is the length, $\lambda$ the thermal conductivity and $S$ the section. $\lambda$ has been estimated using the works of Cheng et al. [9], by estimating the core component dimensions and calculating an equivalent thermal conductivity. $R_{i,+}$ and $R_{i,-}$ were calculated this way.

$$
R=\frac{L}{\lambda S}
$$

Heat transfer in the casing: Casing thermal resistances were also calculated using formula (4), using the thermal conductivity of aluminum $\left(327 W \cdot m^{-1} \cdot K^{-1}\right) . R_{s, z 1}, R_{s, z 2}$, $R_{s, y}$ were obtained this way.

Heat transfer outside the casing: Thermal loss resistances from insulated faces to ambient air (Fig. 3b) were calculated as the sum of three terms. For instance, taking $R_{e, x}$ :
- $R_{e, x 1}$ : contact resistance between the cell and the insulating material;

- $R_{e, x 2}$ : conduction through the insulating material;

- $R_{e, x 3}$ : convection between the insulating material and the ambient air.

Using typical values of contact resistance, $R_{e, x 1}$ was found to be negligible compared to $R_{e, x 2}$ and $R_{e, x 3}$ [14]. $R_{e, x 2}$ has been determined using formula (4) and $R_{e, x 3}$ using formula (5). $h$ is a transfer coefficient, which often needs to be experimentally determined to reach a sufficient precision [15]. In our case, convection has a minor impact on $R_{e, x}$ regarding $R_{e, x 2}$. Therefore, we only aimed at determining a realistic value of $h$ [8].

$$
R=\frac{1}{h S}
$$

$R_{e, x}$ was found to be $24.3 K . W^{-1}$ (with $R_{e, x 3}$ being $\left.2.1 K . W^{-1}\right) . R_{e, y}$ and $R_{e, z}$ were calculated the same way.

Heat transfer between terminals and ambient air: Thermal loss resistances from positive and negative terminals to ambient air are identical and were calculated as the sum of two terms. For instance, taking $R_{e,+}$ :

- $R_{e,+1}$ : contact resistance between the positive terminal and the power wire;

- $R_{e,+2}$ : transfer between the end of the power wire and the ambient air;

$R_{e,+1}$ has been calculated using the surfacic conductance of a copper-copper contact under 2 bars: $6.000 \mathrm{~W} . \mathrm{m}^{-1} . K^{-1}$ [14], leading to $1.7 \mathrm{~K} . \mathrm{W}^{-1}$.

$R_{e,+2}$ represents the heat transfer between the wire end and the ambient air. To represent this transfer, the wire has been discretized into 10 elements of $10 \mathrm{~cm}$. Each of them is connected to the 2 next elements by a conduction resistance (calculated using equation (4)). Each element is also connected to ambient air by 2 series thermal resistances : conduction through the insulating material and convection to the ambient air (equation (5)). For a wire longer than $50 \mathrm{~cm}$, the equivalent resistance $R_{e,+2}$ tends to a constant value, being $8.9 \mathrm{~K} . \mathrm{W}^{-1}$. The value of $R_{e, x}$ is so $10.6 \mathrm{~K} . \mathrm{W}^{-1}$.

Cell and cooling system contact: Finally, the interfacial resistance $R_{e, b o t}$, being the last unknown parameter, has been determined through an identification algorithm. The cell has been heated by a square current in the experimental setup shown by figure $3 a$ (same solicitation as for the heat capacity determination), with a thermocouple on its face in $\mathrm{x}$ direction. Its temperature has been recorded at the thermal steady state. Then, the value of $R_{e, b o t}$ has been adjusted for the model to fit the experimental data. It was found to be $1.8 \mathrm{~K} . \mathrm{W}^{-1}$.

\section{EXPERIMENTAL VALIDATION}

The cell, as shown by figure $3 \mathrm{a}$, was packed with insulating materials on its upper and lateral faces. The cell bottom was connected to a temperature-regulated system set to $15.5^{\circ} \mathrm{C}$ and the ambient air was at $22^{\circ} \mathrm{C}$. The cell was at a thermal steady state before being: 
- fully discharged in $30 \mathrm{~min}(2 \mathrm{C}$ current);

- $\quad$ rested for 30 minutes;

- fully charged in 2 hours (C/2 current, CCCV method);

- $\quad$ rested for 30 minutes.

Voltage and current profiles were recorded (Bio-Logic HCP1005 ) to evaluate the heat generation rate (equation (1)) (Fig. 4a) and was then used to simulate the cell thermal behavior. Temperature on its face in $\mathrm{x}$ direction was recorded and compared to simulations (Fig. 4b).

It can be seen that much more heat is generated during the discharge than during the charge, because of the lower charging current. The heat generated while discharging the cell is mostly due to electrical losses (irreversible heat). Two heat peaks can be observed at the beginning and at the end of the discharge, due to the internal resistance increase near $0 \%$ and $100 \%$ SoC. The discharge-end heat-peak is even more increased by the reversible heat generation, because chemical reactions are exothermic near $0 \% \mathrm{SoC}$ for a discharge current (these reactions will so be endothermic and will absorb heat under a charge current).

During the charging step, both reversible and irreversible heats contributes significantly to the total generated heat. At the beginning of the charge, a heat absorption can be observed : meaning that the heat consumed by chemical reactions is superior to the heat generated by electrical losses. At the end of the charge, the heat peak and the following gradual decrease are due to the CCCV charging method.

Figure $4 \mathrm{~b}$ represents the temperature evolutions of the:

- $T_{\text {surf }}$ : face in $\mathrm{x}$ direction (measured and calculated);

- $T_{\text {core }}$ : cell core (measured);.

- $T_{\text {bottom }}$ : cell bottom face (measured);

- $T_{\text {air }}$ : ambient air (measured);

- $T_{\text {cooling }}$ : cooling system (set);

The model we used for heat generation estimation seems to give good results, as the temperature evolutions are in accordance with measurements. The thermal steady state between $2 h$ and $3 h$ is also well represented, reflecting the correctness of thermal resistances. The precision of our model during this test is better than $1^{\circ} \mathrm{C}$.

\section{CONCLUSION AND DISCUSSIONS}

A lumped thermal model for a large prismatic lithium cell has been presented. The proposed model aims to represent the thermal behavior of a pack central cell. The proposed model has been experimentally validated, is able to represent typical thermal phenomena and gives reliable information on cell key temperatures.

Simulation shows a difference of $3^{\circ} \mathrm{C}$ between $T_{\text {surf }}$ et $T_{\text {core }}$. This is quite small, whereas the cell is well-cooled and is so able to stay in acceptable temperature ranges. As for the casing, $T_{\text {bottom }}$ is about $20^{\circ} \mathrm{C}$ colder than $T_{\text {surf }}$ at the end of the discharge : this strong difference shows that cells is indeed cooled by its bottom face through its casing. Finally, $T_{\text {bottom }}$
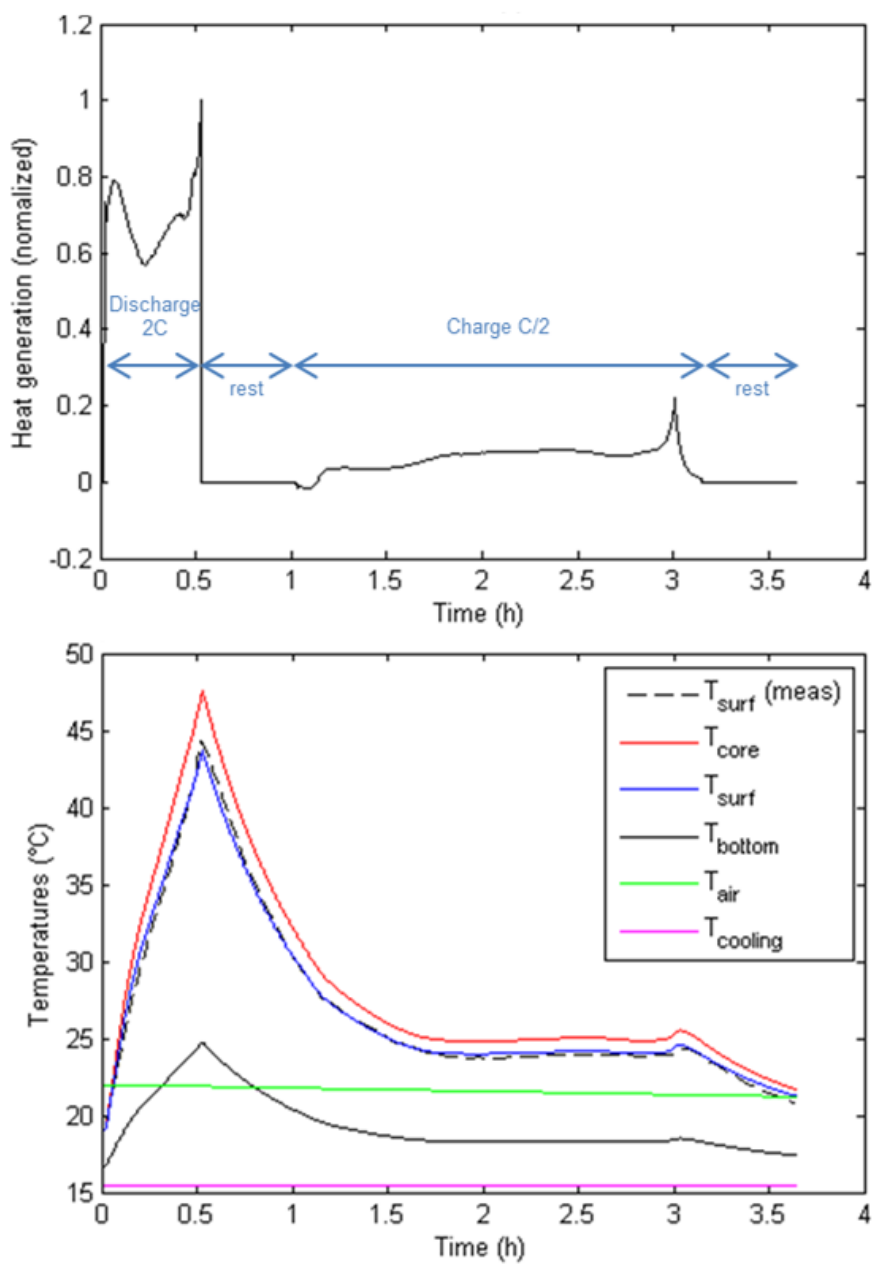

Figure 4. (a) Heat generated and (b) temperature simulations compared to measures for a discharge-charge cycle.

reaches a $10^{\circ} \mathrm{C}$ difference with the cooling system, only due to the interfacial thermal resistance. This confirms the sensibility of the system cooling-performances to this interface and, as a result, strengthens the dependence of the simulation quality to this resistance determination.

The model is well-suited for embedded applications such as a BMS or for off line applications such as a pack thermaldesign tool. It is also suitable for larger model (pack model) as several cell models can easily be interconnected. To do so, thermal transfers between cells and with the pack environment should be characterized, as well as the influence of these transfers of cell thermal behaviors.

\section{REFERENCES}

[1] Said Al Hallaj, Hossein Maleki, Jong-Sung Hong, and J. Robert Selman. Thermal modeling and design considerations of lithium-ion batteries. Journal of Power Sources, 83(1-2):1-8, 1999.

[2] Christophe Forgez, Dinh Vinh Do, Guy Friedrich, Mathieu Morcrette, and Charles Delacourt. Thermal modeling of a cylindrical LiFePO4/graphite lithium-ion battery. Journal of Power Sources, 195(9):2961-2968, 2010.

[3] Kazuo Onda, Takamasa Ohshima, Masato Nakayama, Kenichi Fukuda, and Takuto Araki. Thermal behavior of small lithium-ion battery 
during rapid charge and discharge cycles. Journal of Power Sources, 158(1):535-542, 2006.

[4] Andrey Smyshlyaev, Miroslav Krstic, Nalin Chaturvedi, Jasim Ahmed, and Aleksandar Kojic. PDE model for thermal dynamics of a large Li-ion battery pack. American Control Conference (ACC), IEEE, pages 959-964, 2011.

[5] Xiao Hu, Saeed Asgari, Shaohua Lin, Scott Stanton, and Wenyu Lian. A linear parameter-varying model for HEV/EV battery thermal modeling. Energy Conversion Congress and Exposition (ECCE), IEEE, pages 1643-1649, 2012.

[6] Andrei Pruteanu, Bogdana Valeria Florean, Georgiana Maria Moraru, and Romeo Cristian Ciobanu. Development of a thermal simulation and testing model for a superior lithium-ion-polymer battery. Optimization of Electrical and Electronic Equipment (OPTIM), IEEE, pages 947952, 2012.

[7] Chakib Alaoui. Solid-State Thermal Management for Lithium-Ion EV Batteries. Vehicular Technology, IEEE Transactions on, 62(1):98-107, 2013.

[8] K\&K Associates. Thermal Network Modeling Handbook. K\&K Associates, 1999.

[9] Cheng Lin, Ke Chen, and Fengchun Sun. Research on thermo-physical properties identification and thermal analysis of EV Li-ion battery. Vehicle Power and Propulsion Conference (VPPC), IEEE, pages 16431648, 2009.

[10] Evgenij Barsoukov, Jee Hwan Jang, and Hosull Lee. Thermal impedance spectroscopy for $\mathrm{Li}$-ion batteries using heat-pulse response analysis. Journal of Power Sources, 109(2):313-320, 2002.

[11] Ralph E. Williford, Vilayanur V. Viswanathan, and Ji-Guang Zhang. Effects of entropy changes in anodes and cathodes on the thermal behavior of lithium ion batteries. Journal of Power Sources, 189(1):101107, 2009.

[12] Wolfgang Dreyer, Janko Jamnik, Clemens Guhlke, Robert Huth, Joze Moskon, and Miran Gaberscek. The thermodynamic origin of hysteresis in insertion batteries. Nature Materials, 9:1-6, 2010.

[13] S.Kar Chowdhury and Prem Kr. Baski. A simple lumped parameter thermal model for electrical machine of TEFC design. Power Electronics, Drives and Energy Systems (PEDES) \& Power India, IEEE, pages 1-7, 2010.

[14] Yves Bertin. Refroidissement des machines électriques tournantes. Techniques de l'ingénieur, pages 1-22, 1999.

[15] Xinfan Lin, Hector E. Perez, Jason B. Siegel, Anna G. Stefanopoulou, Yonghua Li, R. Dyche Anderson, Yi Ding, and Matthew P. Castanier. Online Parameterization of Lumped Thermal Dynamics in Cylindrical Lithium Ion Batteries for Core Temperature Estimation and Health Monitoring. Control Systems Technology, IEEE Transactions on, PP(99):1-11, 2012. 